\title{
The effect of dust storms on the atmospheric microbiome in the Eastern
}

\section{Mediterranean}

\section{Supporting Information}

\author{
Yinon Mazar ${ }^{1}$, Eddie Cytryn ${ }^{2}$, Yigal Erel ${ }^{3}$, Yinon Rudich ${ }^{1, *}$ \\ ${ }^{1}$ Department of Earth and Planetary Sciences, Weizmann Institute of Science, Rehovot \\ 76100, Israel \\ ${ }^{2}$ Institute of Soil, Water and Environmental Sciences, The Volcani Center, Agriculture \\ Research Organization, Bet Dagan, Israel \\ ${ }^{3}$ The Fredy and Nadine Herrmann Institute of Earth Sciences, The Hebrew University of \\ Jerusalem, Israel \\ Corresponding author: *E-mail: Yinon.rudich@weizmann.ac.il
}

\section{Table of Contents}

Table S1. Air quality and meteorological parameters..........................................................S2

Table S2. Sources used of for the sequencing data.................................................................

Table S3. Taxa displaying decreased or increased abundance during dust events. .....................S3

Figure S1. Schematic diagram of the pNORM reference plasmid.............................................S4

Figure S2. PM10 concnetrations during the two sampling periods ............................................S5

Figure S3. Atmospheric concentrations of dust and marine tracer elements ...............................S5

Figure S4. Back trajectory of a typical air mass during a dust event ........................................S6

Figure S5. Average relative abundance of prominent defined classes in the collected atmospheric samples S7 
Table S1. Air quality and meteorological parameters measured during sampling events. Overall 16 ambient day and 10 dust day were sampled.

\begin{tabular}{|c|c|c|c|c|c|c|}
\hline $\begin{array}{l}\text { Sampling } \\
\text { Date }\end{array}$ & $\begin{array}{l}\text { Sampling } \\
\text { time }(\mathrm{h})\end{array}$ & Notes & $\begin{array}{l}\text { PM10 average } \\
{\left[\mu \mathrm{g} / \mathrm{m}^{\wedge} 3\right]}\end{array}$ & $\begin{array}{l}\text { PM10 Maximum } \\
\text { value }\left[\mu \mathrm{g} / \mathrm{m}^{\wedge} 3\right]\end{array}$ & $\begin{array}{l}\text { Fine mode fraction } \\
(550 \mathrm{~nm})^{*}\end{array}$ & $\begin{array}{l}\text { Dust } \\
\text { origin }\end{array}$ \\
\hline $19,20.2 .14$ & 24 & Dust-free day & 43.6 & 63.2 & 0.86 & \\
\hline $23,24.2 .14$ & 24 & $\begin{array}{l}\text { Days after } \\
\text { minor event }\end{array}$ & 77.4 & 105.5 & 0.42 & $\begin{array}{l}\text { North } \\
\text { Africa }\end{array}$ \\
\hline $26,27.2 .14$ & 24 & Dust-free day & 20.7 & 35.3 & 0.46 & \\
\hline 3.3 .14 & & $\begin{array}{l}\text { Major dust } \\
\text { event }\end{array}$ & 450.9 & 844.2 & 0.18 & $\begin{array}{l}\text { North } \\
\text { Africa }\end{array}$ \\
\hline $3,4.3 .14$ & 9.167 & $\begin{array}{l}\text { Days after } \\
\text { major event }\end{array}$ & 219.5 & 465.7 & 0.15 & \\
\hline $5,6.3 .14$ & 24 & $\begin{array}{l}\text { Days after } \\
\text { major event }\end{array}$ & 150.8 & 270.9 & 0.19 & \\
\hline $6,7.3 .14$ & 24 & $\begin{array}{l}\text { Days after } \\
\text { major event }\end{array}$ & 137.9 & 324.4 & 0.12 & \\
\hline $10,11.3 .14$ & 24 & Dust-free day & 21.4 & 39.3 & 0.51 & \\
\hline $11,12.3 .14$ & 24 & Rain & 14.9 & 49 & 0.66 & \\
\hline $3,4.2 .15$ & 24 & $\begin{array}{l}\text { Minor dust } \\
\text { event }\end{array}$ & 121.2 & 256 & 0.38 & $\begin{array}{l}\text { North } \\
\text { Africa }\end{array}$ \\
\hline $4,5.2 .15$ & 24 & $\begin{array}{l}\text { Days after } \\
\text { minor event }\end{array}$ & 75.5 & 156 & 0.32 & \\
\hline 6.2 .15 & 10.5 & $\begin{array}{l}\text { Days after } \\
\text { minor event }\end{array}$ & 76.7 & 315 & 0.47 & \\
\hline $9,10.2 .15$ & 24 & $\begin{array}{l}\text { Days after } \\
\text { minor event }\end{array}$ & 60.5 & 93 & 0.29 & \\
\hline $10,11.2 .15$ & 15.567 & $\begin{array}{l}\text { Major dust } \\
\text { event }\end{array}$ & 1100.2 & 2938 & $0.09 * *$ & $\begin{array}{l}\text { North } \\
\text { Africa }\end{array}$ \\
\hline 11.2 .15 & 6 & $\begin{array}{l}\text { Major dust } \\
\text { event }\end{array}$ & 1231.9 & 1619 & $0.02 * *$ & $\begin{array}{l}\text { North } \\
\text { Africa }\end{array}$ \\
\hline $16,17.2 .15$ & 24 & Dust-free day & 37.1 & 102 & 0.79 & \\
\hline $22,23.2 .15$ & 24 & Dust-free day & 33.8 & 86 & 0.29 & \\
\hline $24,25.2 .15$ & 24 & Dust-free day & 58.2 & 169 & 0.14 & \\
\hline $1,2.3 .15$ & 24 & $\begin{array}{l}\text { Minor dust } \\
\text { event }\end{array}$ & 138.4 & 258 & 0.14 & $\begin{array}{l}\text { North } \\
\text { Africa }\end{array}$ \\
\hline $8,9.3 .15$ & 24 & $\begin{array}{l}\text { Minor dust } \\
\text { event }\end{array}$ & 114.8 & 213 & 0.26 & $\begin{array}{l}\text { North } \\
\text { Africa }\end{array}$ \\
\hline $10,11.3 .15$ & 24 & $\begin{array}{l}\text { Days after } \\
\text { minor event }\end{array}$ & 74 & 86.7 & 0.33 & \\
\hline $12,13.3 \cdot 15$ & 24 & Dust-free day & 30.9 & 84 & 0.55 & \\
\hline $15,16.3 .15$ & 24 & Dust-free day & 24.5 & 49 & 0.72 & \\
\hline $22,23.3 .15$ & 24 & Dust-free day & 34.8 & 70 & 0.51 & \\
\hline $27,28.3 .15$ & 24 & $\begin{array}{l}\text { Minor dust } \\
\text { event }\end{array}$ & 97.1 & 227 & 0.24 & $\begin{array}{l}\text { North } \\
\text { Africa }\end{array}$ \\
\hline $28,29.3 .15$ & 24 & $\begin{array}{l}\text { Days after } \\
\text { minor event }\end{array}$ & 69.3 & 127 & 0.26 & \\
\hline
\end{tabular}

*The 2014 Aeronet data was obtained from the Nes-Ziona station, located nearby the sampling location. 2015 data was obtained from the Aeronet station in Sede-Boker because the Nes-Ziona station was not operational during the sampling period. Comparison of data from the two stations on overlapping dates shows the same trend of AOD measurements at both stations. Data was collected on the 1.5 quality level, which provides screening of clouds. 
** Due to lack of data on the 1.5 quality levels on these dates, non-screened data of quality level 1 was used.

Table S2. Sources used of for the sequencing data.

\begin{tabular}{lllll}
\hline Biom & Environment & Source & Researcher & Description \\
\hline Human & Feces & QIITA & Rob Knight & American gut microbiome project \\
Human & Skin & QIITA & Rob Knight & American gut microbiome project \\
Human & Oral Cavity & QIITA & Rob Knight & American gut microbiome project \\
Soil & Desert & MG-RAST & Cheryl Kuske & Desert soil from science focus area, Nevada, \\
& & & & USA \\
Soil-local & Rendzina & collaborators & Edouard Jurkevitch & Local soils from Gimzo area, Israel \\
Soil-local & Sand & collaborators & Edouard Jurkevitch & Local soils from Gimzo area, Israel \\
Soil-local & Terrarossa & collaborators & Edouard Jurkevitch & Local soils from Gimzo area, Israel \\
Agriculture- & Poultry & Co-author & Eddie Cytryn & Litter and airborne material from poultry facility, \\
local & & & & Israel. \\
Sea & Sea & MG-RAST & Stephen Techtmann & Eastern Mediterranean near shore, Egypt \\
\hline
\end{tabular}

Table S3: Taxa displaying decreased or increased abundance during dust events.

\begin{tabular}{|c|c|c|}
\hline Taxonomic level & Abundance significantly increased during dust event & Abundance significantly decreased during dust event \\
\hline Phylum & $\begin{array}{l}\text { Actinobacteria, Bacteroidetes, Chloroflexi, } \\
\text { Gemmatimonadetes, [Thermi] }\end{array}$ & Firmicutes \\
\hline Class & $\begin{array}{l}\text { Acidimicrobiia, Actinobacteria, } \\
\text { Alphaproteobacteria, Chloroflexi, Cytophagia, } \\
\text { Deinococci, Gemm-3, Gemmatimonadetes, } \\
\text { Rubrobacteria, [Saprospirae], Thermoleophilia, } \\
\text { Thermomicrobia }\end{array}$ & Bacilli, Bacteroidia, Clostridia, Gammaproteobacteria \\
\hline Order & $\begin{array}{l}\text { Acidimicrobiales, Actinomycetales, AKIW781, } \\
\text { Cytophagales, Deinococcales, Rhizobiales, } \\
\text { Rhodospirillales, Rubrobacterales, [Saprospirales], } \\
\text { Solirubrobacterales, Sphingomonadales }\end{array}$ & $\begin{array}{l}\text { Bacteroidales, Clostridiales, Lactobacillales, } \\
\text { Pseudomonadales }\end{array}$ \\
\hline Family & $\begin{array}{l}\text { Acetobacteraceae, Bacillaceae, Bradyrhizobiaceae, } \\
\text { Cellulomonadaceae, Chitinophagaceae, } \\
\text { Cytophagaceae, Frankiaceae, Geodermatophilaceae, } \\
\text { Micrococcaceae, Nocardioidaceae, Planococcaceae, } \\
\text { Rubrobacteraceae, Solirubrobacteraceae, } \\
\text { Sphingomonadacea, Sporichthyaceae, } \\
\text { Streptomycetaceae, } \\
\text { Trueperaceae }\end{array}$ & $\begin{array}{l}\text { Brevibacteriaceae, Clostridiaceae, Corynebacteriaceae, } \\
\text { Dermabacteraceae, Lachnospiraceae, Lactobacillaceae, } \\
\text { Microbacteriaceae, Moraxellaceae, Ruminococcaceae, } \\
\text { Staphylococcaceae }\end{array}$ \\
\hline Genus & $\begin{array}{l}\text { Adhaeribacter, Arthrobacter, Bacillus, Balneimonas, } \\
\text { Cellulomonas, Geodermatophilus, Pontibacter, } \\
\text { Rubellimicrobium, Rubrobacter, Sporosarcina }\end{array}$ & $\begin{array}{l}\text { Acinetobacter, Brachybacterium, Brevibacterium, } \\
\text { Corynebacterium, Lactobacillus, Paracoccus, }\end{array}$ \\
\hline
\end{tabular}




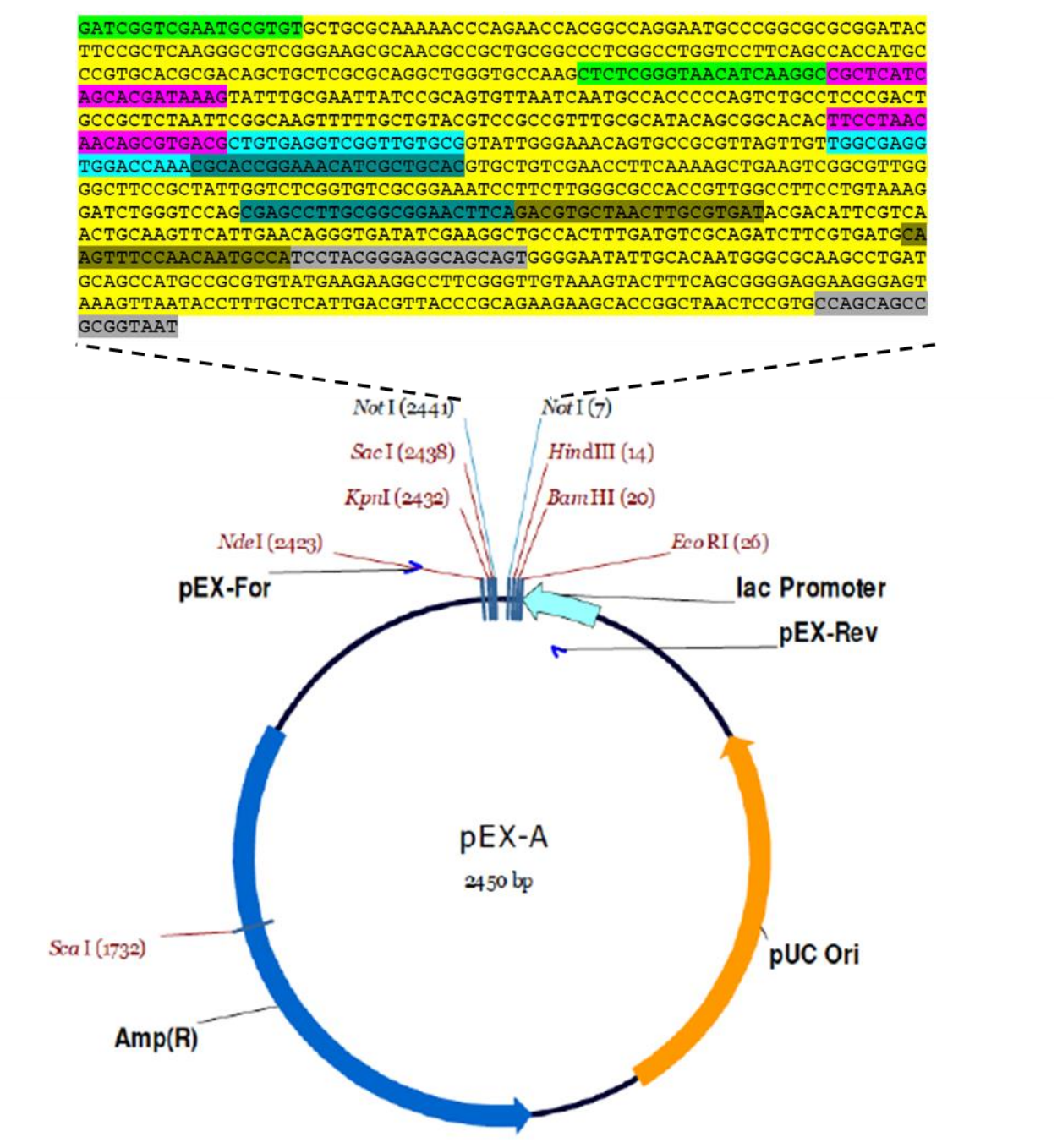

Figure S1. Schematic diagram of the pNORM reference plasmid. The plasmid was constructed by ligating a 892 bp insert sequence (shown above in yellow highlight) containing amplification sites for seven target genes (see below), into a pEX-A2 (pUC derivative) backbone plasmid (circular map below). Amplification sites of the target genes are as follows- Green: intI1 (196bp); Purple: ctxM-32 (156bp); Cyan: vanA (65bp); Blue sul1 (162bp); Gold: qnrS1 (118bp); Grey: 16S rRNA (195bp) and Red: blaTEM (113bp). The plasmid was constructed by Christophe Merlin (LCPME, Nancy, France) and synthesized by Eurofins Scientific. 


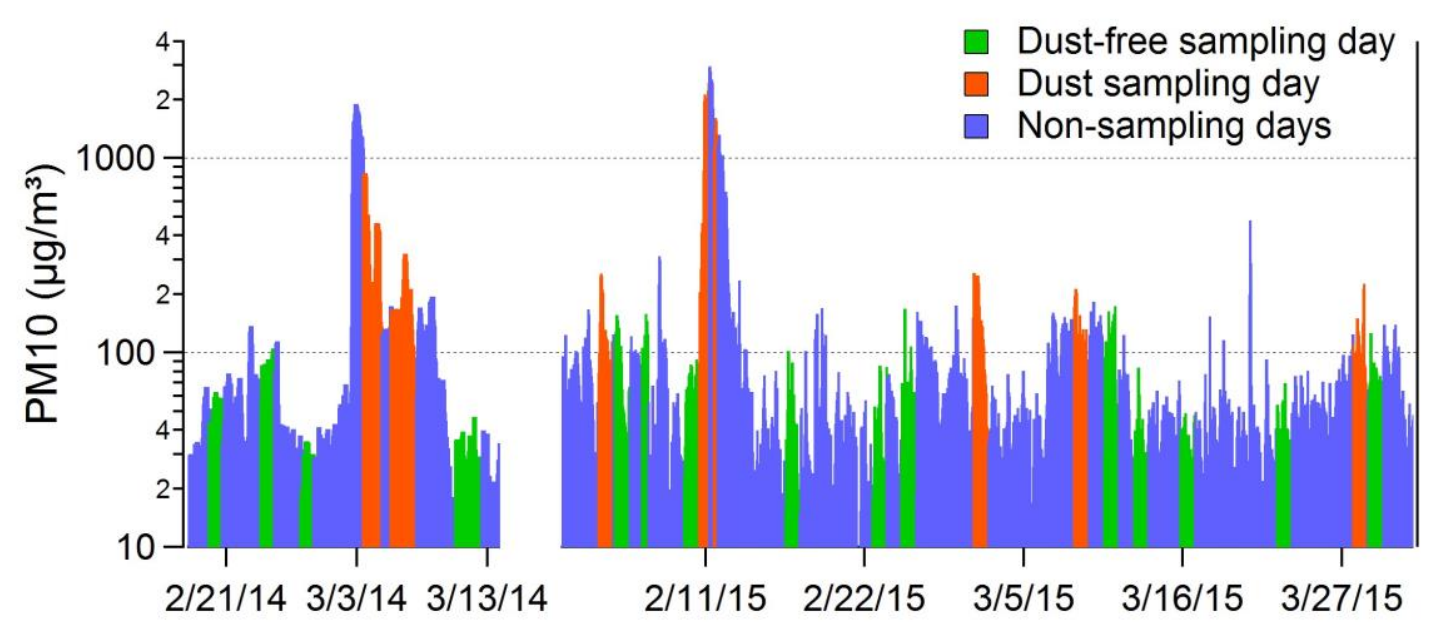

Figure S2. PM10 concnetrations during the two sampling periods as measured in a monitor station in Rehovot, Israel. Sampling days are marked by orange (dust days) and green (dust-free days).
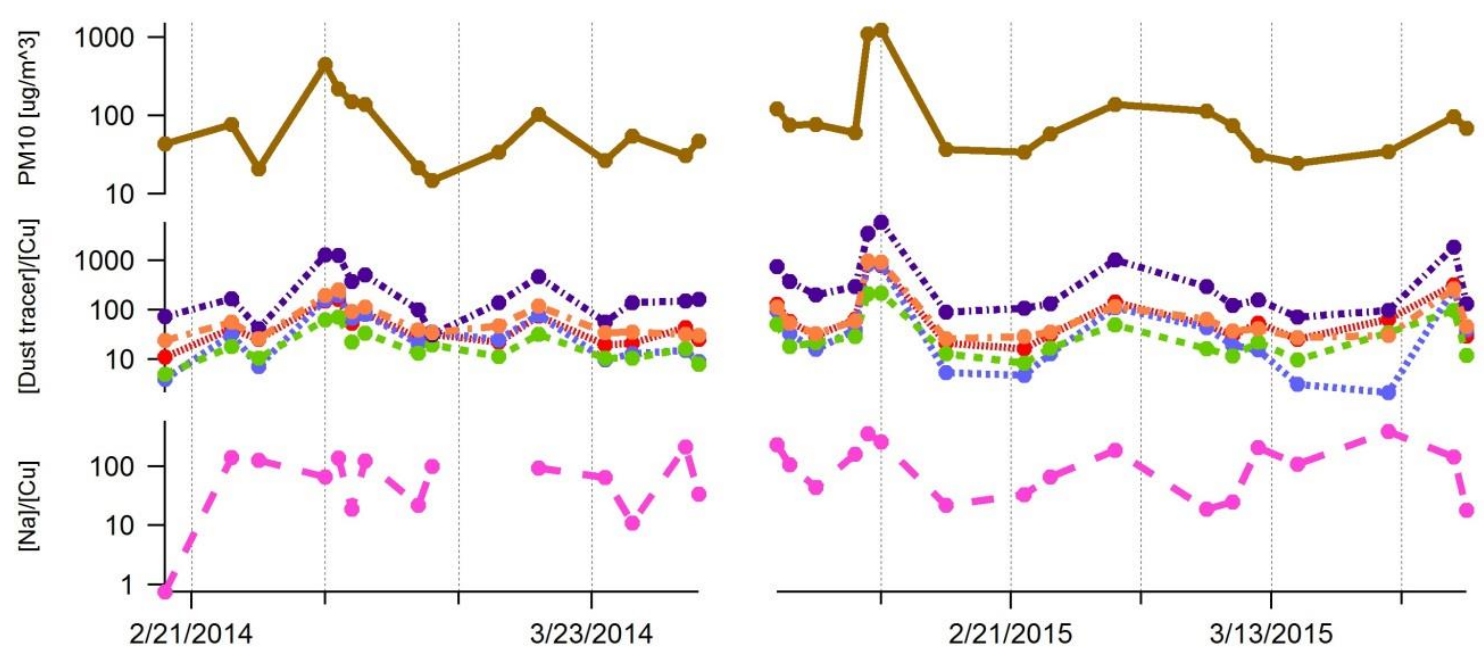

Figure S3. Atmospheric concentrations of dust and marine tracer elements normalized by the atmospheric concentration of $\mathrm{Cu}$, compared to the PM10 levels on the same days. 


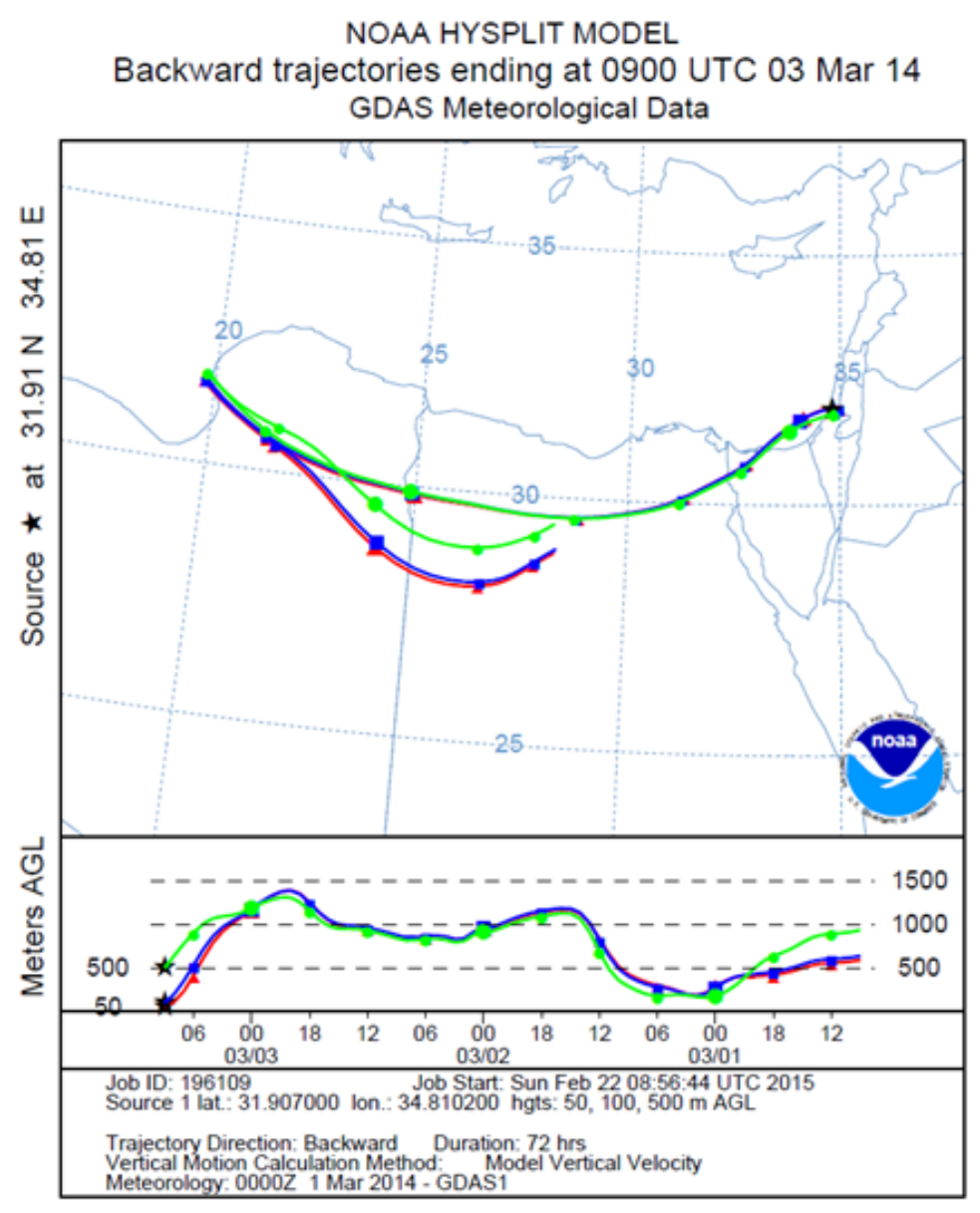

Figure S4. Back trajectory of a typical air mass during a dust event, showing that the airmass did not pass over sea. 

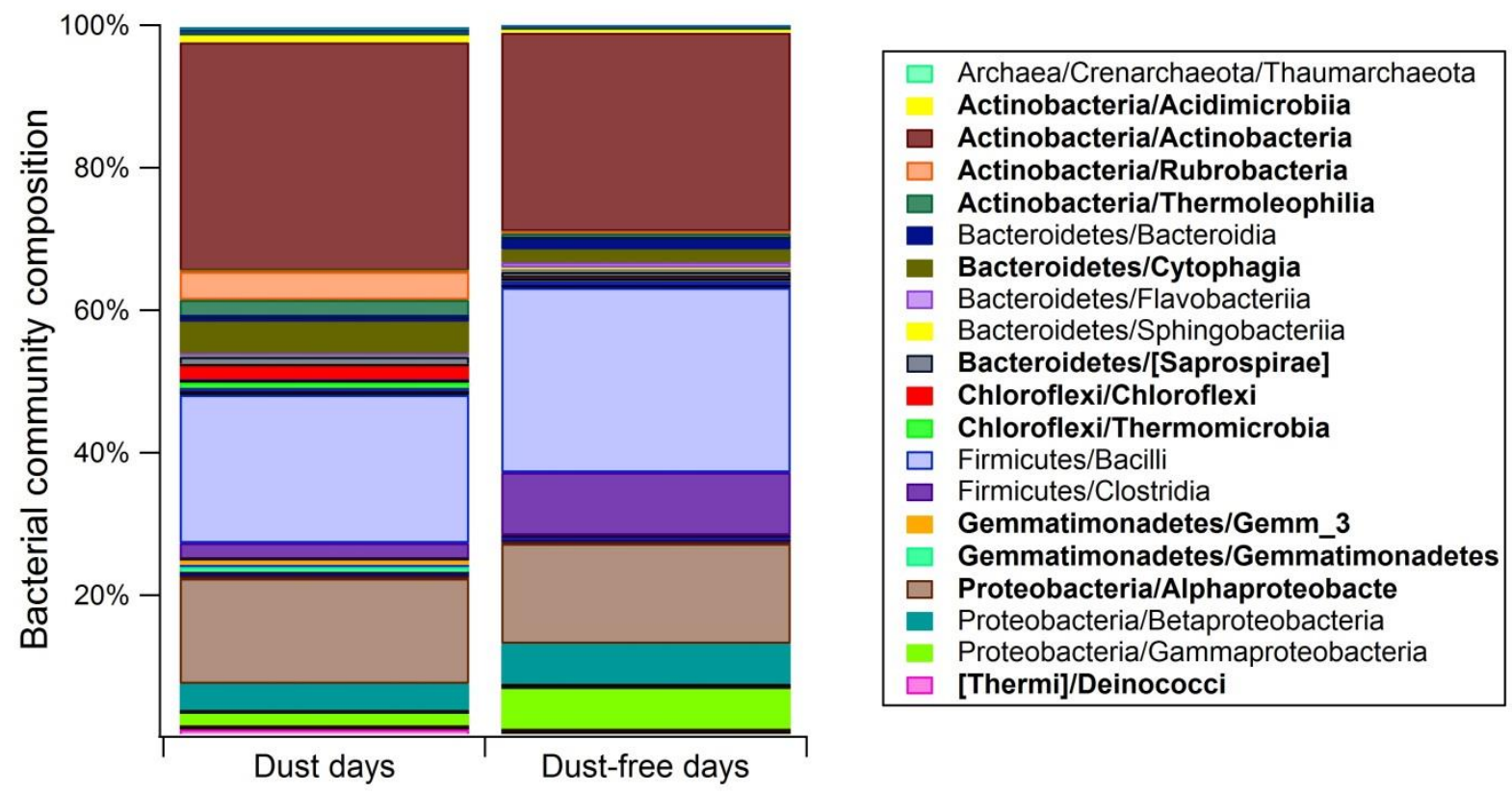

Figure S5. Average relative abundance of prominent defined classes in the collected atmospheric samples. Classes with significantly higher relative abundance $(\mathrm{p}<0.05)$ in dust events are marked in bold. 